\title{
SAÚDE, INTEGRALIDADE E CUIDADO: CONCEPÇÕES DE GESTORES DE UNIDADES BÁSICAS DE SAÚDE
}

\author{
Janaína da Câmara Zambelli', Pedro Henrique Brito da Silva², Nelson Filice de Barros ${ }^{3}$ e \\ Ellen Synthia Fernades de Oliveira ${ }^{4}$ \\ 1,2,3,4 Universidade Federal de Goiás, Brasil. ${ }^{1}$ jcsateles@gmail.com, ${ }^{2}$ pedrobryto@gmail.com, ${ }^{4}$ ellen.synthia@gmail.com \\ ${ }^{3}$ Universidade Estadual de Campinas, Brasil. nelsonfilicel@gmail.com
}

\begin{abstract}
Resumo. As Práticas Integrativas e Complementares (PIC) constituem como significativo instrumento para ampliar o acesso como para a qualificação dos serviços, numa perspectiva da integralidade da atenção à saúde. Este estudo teve como objetivo analisar as concepções de saúde, integralidade e cuidado para o gestor de Unidade Básica de Saúde que não oferta ou deixou de ofertar as práticas integrativas e complementares em uma região metropolitana. Estudo descritivo exploratório de abordagem qualitativa, realizado entre os meses de novembro e dezembro de 2018, com 24 gestores de 16 municípios. Os dados foram coletados por meio de entrevistas semiestruturada, que foram gravadas, transcritas e analisadas tematicamente, com o auxílio do software Nvivo 11. Da análise dos dados emergiram duas categorias: Concepções dos gestores acerca de saúde e Concepções dos gestores sobre integralidade nas PIC. Conclui-se que os gestores de serviços de APS na Região Metropolitana de Goiânia demonstram que ainda persiste a reprodução de ações e práticas centradas no modelo biomédico, embora os gestores reconheçam suas limitações. São fatores como esses que dificultam a implementação e expansão de novos modelos de cuidado, como as PIC.
\end{abstract}

Palavras-chave: Terapias Complementares; Integralidade em Saúde; Gestão em Saúde; Pesquisa Qualitativa.

\section{HEALTH, INTEGRALITY AND CARE IN INTEGRATIVE AND COMPLEMENTARY PRACTICES: CONCEPTIONS OF MANAGERS OF BASIC HEALTH UNITS}

\begin{abstract}
Integrative and Complementary Practices (ICP) are a significant tool both for broadening access to services and for qualifying them from the perspective of integral health care. The objective of this study was to analyze the conceptions of health, integrality, and care for the manager of the Basic Health Unit who does not offer or no longer offers the integrative and complementary practices in a metropolitan region. Descriptive exploratory study of qualitative approach carried out between the months of November and December 2018, with 24 managers from 16 municipalities. The data were collected through semi-structured interviews, which were recorded, transcribed and thematically analyzed, with the help of Nvivo 11 software. Two categories emerged from the data analysis: Managers' Conceptions about health and Managers' Conceptions about integrality in ICPs. It is concluded that managers of PHC services in the Metropolitan Region of Goiânia demonstrate that the reproduction of actions and practices centered on the biomedical model still persists, although managers recognize its limitations. It is factors such as these that make it difficult to implement and expand new models of care, such as PIC.
\end{abstract}

Keywords: Complementary Therapies; Integrality in Health; Health Management; Qualitative Research.

\section{INTRODUÇÃO}

A Organização Mundial da Saúde (OMS), em 1978, conceitua a saúde como um completo bem-estar, de modo que o processo saúde-doença vai se relacionando com a qualidade de vida, vida saudável e estilo de vida. Essa concepção de saúde sinaliza a natureza 
integradora da saúde, incluindo além do aspecto biológico, a dimensão social como também a psicológica. Tal visão foi elaborada na Conferência Internacional sobre Cuidados Primários de Saúde, realizada em 1978, em Alma-Ata, que culminou na Declaração de Alma-Ata, reafirmando a saúde como direito humano fundamental. Nessa conferência, também se recomendou o estabelecimento de políticas nacionais de saúde apoiadas no uso de recursos da medicina tradicional pelos sistemas nacionais de saúde, como fonte de cuidado para melhorar e aprimorar a saúde da população (OMS, 2002).

Nesse sentido, essas recomendações representaram o ponto de partida para as Conferências Internacionais sobre Promoção da Saúde. A Primeira Conferência Internacional Sobre Promoção da Saúde, em 1986, em Ottawa, Canadá, norteia-se por um conceito de saúde ampliado, de modo a envolver condicionantes e determinantes do processo saúde-doença e não meramente a ausência de doenças (Buss \& Carvalho, 2009).

No Brasil, o debate sobre a inclusão de Práticas Integrativas e Complementares (PIC) no sistema nacional de saúde teve início na década de 1980 , com destaque para a $8^{a}$ Conferência Nacional de Saúde (CNS), em 1986, como também após a criação do Sistema Único de Saúde (SUS), em 1988 (BRASIL, 2006). Nessa conferência, nota-se uma demanda da população brasileira para formulação de políticas orientadas à institucionalização das abordagens terapêuticas integrais e dos recursos nelas utilizados no SUS (Tesser, 2018).

Para atender essa demanda, tal conferência, impulsionada pela Reforma Sanitária, foi apontada como marco legal e institucional das PIC. Entre as várias deliberações, tem-se no relatório final da conferência a seguinte redação (Brasil, 1986, p. 10): "Introdução de práticas alternativas de assistência à saúde no âmbito dos serviços de saúde, possibilitando ao usuário o acesso democrático de escolher a terapêutica preferida".

Como resposta à essa conjuntura, o Ministério da Saúde (MS) aprova, em 2006, a Política Nacional de Práticas Integrativas e Complementares (PNPIC), pela Portaria Ministerial $n^{\circ}$ 971 (Brasil, 2006). Inicialmente, o documento ministerial contemplou cinco PIC, quais sejam: Medicina Tradicional Chinesa/Acupuntura, Fitoterapia, Termalismo e Crenoterapia Social, Homeopatia e a Medicina Antroposófica. Mais recentemente, com a publicação das Portarias $n^{\circ}$ 145/2017, $n^{\circ}$ 849/2017 e $n^{\circ}$ 702/2018, mais 24 práticas, totalizando 29, incluindo, por exemplo, a Arteterapia, o Reiki, a Osteopatia, a Quiropraxia e a Terapia Comunitária (Brasil, 2017). 
Segundo Telesi Júnior (2016), a riqueza das PIC se caracteriza por suas práticas interdisciplinares e linguagens singulares, contrapondo à visão fragmentada do tratamento do usuário em especialidades, o qual acaba por menosprezar o todo que se constitui o ser humano.

Conforme Pagliosa \& Da Rosa (2008), é crescente a necessidade de um trabalho multiprofissional, como também o conhecimento interdisciplinar para enfrentar as necessidades de saúde das pessoas e comunidades, cada vez mais complexas. Logo, urge a criação de competências para o melhor desempenho dos profissionais da saúde, no sentido de que essas habilidades sejam baseadas nos conhecimentos e práticas da saúde coletiva, do entendimento e a participação na construção das políticas públicas e na organização dos serviços de saúde.

Para Rios, Franchi, Silva, Amorim, \& Costa (2007), atualmente, predomina o conhecimento racional e o conhecimento científico entre os profissionais de saúde, de forma que a maioria tende a desmerecer o senso comum, o modo como as pessoas do saber popular vivencia e compreende o mundo. Porém, eles acreditam que esses profissionais, formados sob a ótica do paradigma biomédico, cartesiano, sejam capazes de questionar essa visão fragmentada e hegemônica. Para tanto, há a necessidade de buscar novos modelos capazes de encarar a complexidade da realidade humana, de modo a inserir uma compreensão histórica e crítica da concepção vigente de saúde.

Contudo, embora a oferta das PIC no SUS tenha se constituído significativo instrumento, tanto para ampliar o acesso, como para qualificar o serviço, em uma perspectiva da integralidade da atenção à saúde, elas ainda são desconhecidas pela maioria dos profissionais de saúde (Brasil, 2015). Nesse sentido, este estudo teve como objetivo analisar as concepções de saúde, integralidade e cuidado para o gestor de Unidade Básica de Saúde que não oferta ou deixou de ofertar as práticas integrativas e complementares em uma região metropolitana.

\section{METODOLOGIA}

Estudo descritivo exploratório de abordagem qualitativa, realizado entre os meses de novembro e dezembro de 2018. O presente estudo é um recorte da dissertação de mestrado, ainda em andamento, sobre "Práticas Integrativas e Complementares na Atenção Primária à Saúde: percepções de gestores sobre os serviços na Região Metropolitana de Goiânia". 
A pesquisa é, além da construção de um conhecimento, um procedimento de aprendizagem, sendo um princípio científico e educativo. Como o processo de construção e desenvolvimento de um conhecimento a respeito de algo, a pesquisa tem como objetivo, por meio da utilização de métodos científicos, resolver problemas e solucionar dúvidas. Então, partindo das interrogações formuladas em relação a fatos ainda obscuros, os estudos qualitativos se justificam, pois são adequados para a compreensão de fenômenos dentro do seu contexto. Estabelece ligações entre conceitos, representações, crenças e comportamentos, além de revelar insights críticos que ajudam a lidar com as deficiências, como por exemplo, do sistema de saúde (Toquette \& Minayo, 2016).

O presente estudo traz como objeto de estudo os 24 serviços de Atenção Primária à Saúde da Região Metropolitana de Goiânia que não ofertam ou deixaram de ofertar as Práticas Integrativas e Complementares. Os participantes do estudo foram os gestores dos serviços de APS que não ofertam ou deixaram de ofertar as PIC, totalizando 24 gestores responsáveis por 24 Unidades Básica de Saúde.

Neste estudo, tomaremos como base o gestor e/ou gerente dos serviços de APS que assume o gerenciamento de unidade básica de saúde, de acordo com o recomendado pela Política Nacional de Atenção Básica 2017 (PNAB), que traz o papel do gerente de atenção básica, orientando a sua inserção na equipe, por meio de apoio financeiro federal (Brasil, 2017).

Foram incluídos os gestores de ambos os sexos que gerenciavam os serviços de APS que não ofertavam ou deixaram de ofertar PIC no momento do estudo, com cadastro ativo no Cadastro Nacional de Estabelecimento de Saúde ${ }^{1}$ (CNES), com no mínimo seis meses de atuação na unidade pesquisada. Foram excluídos os gestores que não estavam desenvolvendo suas atividades no período da coleta de dados por motivos de licença médica, licença maternidade e/ou paternidade ou férias.

A coleta de dados ocorreu no período de novembro a dezembro de 2018. Após o cumprimento dos preceitos éticos, os gestores foram convidados a participar do estudo pela pesquisadora, por meio de contato telefônico. Diante do aceite, foi agendado o dia e o horário da entrevista no local de trabalho do gestor. Posteriormente, foi realizada a visita reforçando os objetivos do estudo, garantindo o anonimato e sigilo das informações. Diante

\footnotetext{
${ }^{1}$ CNES é base para operacionalizar os Sistemas de Informações em Saúde. Dispõe de um vasto conteúdo de informações, proporcionando ao gestor conhecer a rede assistencial e sua potencialidade, imprescindíveis nos processos de planejamento em saúde, regulação, avaliação, controle e auditoria, bem como dar visibilidade ao controle social para o melhor desempenho de suas funções (BRASIL, 2006).
} 
da concordância, foi apresentado o Termo de Consentimento Livre e Esclarecido (TCLE), para ser lido e assinado, em duas vias.

Nesse sentido, a entrevista foi dirigida pelas seguintes questões norteadoras: "Para você, o que é saúde? Para você, o que significa integralidade e cuidado na Atenção Primária à Saúde? Qual o maior desafio para se alcançar essa integralidade neste serviço de saúde?”.

Após a transcrição das entrevistas, os dados foram processados e analisados por meio da técnica de Análise de Conteúdo Temática proposta por Bardin (2016). Para tanto, foi utilizado o software NVivo ${ }^{\circledR}$ versão 11 (QRS International PTY, Doncaster, Austrália), que consiste em um software de análise de textos e áudios, por exemplo, de modo a possibilitar a edição, visualização, interligação e organização dos documentos, de maneira que o pesquisador cria categorias, questionando os dados e respondendo ao seu objetivo de estudo que consiste em um sistema de indexação e categorização de dados não estruturados, de modo a proporcionar a descoberta e exploração dos sentidos das informações (Mozzato, Grzybovski, \& Teixeira, 2016).

Conforme a técnica de Análise de dados, definida por Bardin (2016) buscou-se a análise em diferentes etapas: pré-análise, exploração do material ou codificação, tratamento dos resultados obtidos, inferência e interpretação.

O presente estudo foi desenvolvido respeitando os requisitos éticos de pesquisa com seres humanos disciplinados nas Resoluções n 466/2012 e 510/2016 do Conselho Nacional de Saúde (CNS). Para tanto, foi submetido e aprovado pelo Comitê de Ética em Pesquisa da Universidade Federal de Goiás (UFG), mediante o parecer consubstanciado n 2977041 , resguardando a todos os participantes o total anonimato, apresentando os riscos e benefícios do estudo.

\section{RESULTADOS}

Os gestores pesquisados atuam em 16 municípios de pequeno e médio porte, com exceção de Goiânia. Nesses serviços, foi possível observar que ainda existe uma forte fragilidade quando se considera o sentido básico do que é um serviço de saúde. Essa fragilidade vai desde problemas de organização, falta de recursos humanos capacitados, deficiências na estrutura física e escassez de recursos materiais. 
A caracterização do perfil do profissional permitiu verificar que prevaleceu o sexo feminino (87\%), com hegemonia dos gestores enfermeiros (58\%). A média de idade dos 24 gestores entrevistados era de 31 anos. No que se refere ao tempo médio de serviço na APS, observou-se que foi de 26 meses. Além disso, constatou-se que a maioria era contratado pelo estatuto do servidor público.

A análise dos dados possibilitou o agrupamento das respostas em duas categorias principais, sendo estas: Concepções dos gestores acerca de saúde no contexto das Práticas Integrativas e Complementares e Concepções dos gestores sobre integralidade no contexto das Práticas Integrativas e Complementares.

\subsection{Concepções dos gestores acerca de saúde}

O propósito dessa categoria é apreciar o aspecto da concepção e identificar a maneira como se faz o direcionamento das ações e práticas nos serviços de saúde, pretendendo o aperfeiçoamento no campo da saúde coletiva, bem como compreender sua relação na implementação da PNPIC e na oferta das PIC.

Ao serem indagados sobre o conceito de saúde e as implicações que este traz ou não ao seu trabalho, os gestores se mostraram esclarecidos e até mesmo estimulados a falar sobre o tema:

"G18) Saúde é tudo de importante. É poder ajudar as pessoas, poder buscar melhorias. Acho que a saúde engloba tudo que o ser humano precisa. Eu amo a saúde."

As concepções, compreensões e sentidos a respeito do que seja saúde são cercados de complexidades. Em virtude dessa definição envolver um campo bastante amplo, torna-se possível que cada pessoa opine a respeito. Foram identificados a partir da análise do material obtido nas entrevistas, conceitos voltados para a qualidade de vida, para o bemestar e também para os Determinantes Sociais da Saúde (DSS). Na perspectiva do gestor, a saúde assume uma versão positiva, como indicam os trechos das entrevistas a seguir:

“G1) [...] saúde é qualidade de vida."

“G3) Bem-estar físico, mental, material também, porque tudo envolve.”

Nos discursos dos participantes pode ser observado que o conceito de saúde preconizado pela OMS perpassa concepções que, apesar da amplitude, ainda se percebe um discurso reducionista, centrado no corpo, a partir da ênfase dada aos comportamentos individuais. 
Do ponto de vista de alguns gestores, a saúde é concebida também com o fazer, com o agir do profissional, relacionando-a, especificamente, a prevenção:

“G12) [...] na unidade básica, de acordo com que a gente trabalha, creio que é a prevenção, orientações aos pacientes. É tratar. É cuidar. É vínculo [...]"

"G17) [...] então, o que eu puder levar de bem-estar e saúde, como também prevenção para o meu paciente, para a minha comunidade, eu levarei."

\subsection{Concepções dos gestores sobre integralidade nas Práticas Integrativas e Complementares}

A inclusão da integralidade ao conceito de saúde ocorreu em vários relatos dos gestores, demonstrando em alguns, uma visão integral do ser humano, como também o inconformismo com o modelo vigente de se perceber a saúde, como se vê nas falas a seguir:

"G10) Saúde é o que não acontece [...]. Saúde é o integral [...]. É atender a pessoa na integralidade, e não só ver a doença em si. É enxergá-la como um todo. Tanto físico, quanto o principal, o emocional."

"G11) Saúde é cuidar de todas as partes da integralidade. Parte física, psicológica, mental, social. Tudo! Familiar também. Isso é saúde. É estar bem com tudo."

Por outro lado, o gestor (G12), expressa em seu relato, uma concepção da integralidade mais direcionada ao nível da relação de trabalho da unidade com os outros níveis de assistência, demonstrando uma visão na perspectiva da articulação do serviço, como se observa em seu relato:

"G12) A gente, aqui, na unidade, consegue atender ao paciente na integralidade [...]. Na verdade, conseguimos em partes, porque não depende só do nosso trabalho [...]. A gente, muito das vezes, precisa encaminhar o paciente [...]. O que depende do município, a gente, até consegue com mais facilidade, mas, às vezes, o município não tem aquele serviço próprio para ofertar. Então, a gente tem que buscar o serviço em outro município, que, no caso, é Goiânia."

Ainda com relação à concepção de integralidade, identifica-se nos discursos um esforço para a melhoria da atenção à saúde dos usuários assistidos, de modo a alcançar à integralidade. Todavia, as narrativas direcionam para a dificuldade de se concretizar plenamente essa integralidade, demonstrando a desintegração na prática do serviço. Um dos gestores diz que:

“G8) A gente tenta sim, mas ainda é algo distante. Ainda existe a forte cultura do atendimento. Alguns gostam e pedem orientações, mas a maioria não tem paciência para ouvir, para mudar o comportamento, mudar o estilo de vida. Assim é difícil." 
Referindo-se a forma como a equipe coloca a integralidade em prática, os entrevistados mostraram, durante as suas falas, haver a hegemonia do modelo biomédico na APS, reforçando a tão discutida fragmentação da atenção, explicitados na seguinte narrativa:

“G18) A questão da integralidade ainda falta muito para acontecer. Falta melhoria. Ainda prevalece o tratamento, sendo o preventivo e a promoção de saúde deixado para segundo plano."

Os participantes relacionaram o cuidado primário à saúde como símbolos do cuidar, do prevenir, e do enxergar o usuário de forma integral, aproximando aos preceitos das práticas integrativas e complementares:

“G4) Proporcionar saúde é o cuidado. É ver o indivíduo. Ver as suas necessidades.”

“G5) Cuidado em saúde é prevenção. Se eu prevenir, eu vou ter uma saúde melhor."

Nesse sentido, percebe-se que os significados atribuídos ao cuidado, à atenção e à integralidade, compreendem também à responsabilização do profissional enquanto cuidador na APS:

“G8) Identificar, tratar bem o paciente. Saber da sua necessidade. Saber do que ele necessita. Muitas vezes, o paciente chega calado. Daí cabe a nós, profissionais, saber perguntar a ele. Saber o que ele tem, né! Principalmente, na Estratégia Saúde da Família, que já é para isso."

\section{DISCUSSÃO}

Para Oliveira e Pereira (2013), a substituição do sistema hegemônico, centrado na assistência curativa, hospitalar e super especializada, que funciona na vertente do interesse econômico e corporativo, por um sistema universal, que busca modelos de atenção que valorizem a integralidade, o cuidado humanizado e a promoção da saúde, depende, em grande parte, do perfil de formação e da prática dos profissionais de saúde. Portanto, o desafio está na quebra da fragmentação do processo de trabalho, que separa o pensar e o fazer desses profissionais, incluindo a integralidade e a humanização das práticas ainda durante a formação.

Considerando a gestão de serviços de saúde na APS, compreende-se que esta constitui lócus de grande relevância ao se considerar o papel estratégico destes espaços, como ordenador da atenção e na coordenação do cuidado em saúde. Com isso, acredita-se que o espaço organizacional que os gestores destes serviços ocupam é de extrema relevância no SUS (Pinto, 2014). 
Embora o acesso aos serviços de atenção primária à saúde tem sido crescente nas últimas décadas, com ampliação da cobertura populacional, pelas equipes de saúde da família em todo território brasileiro, ainda existem desafios históricos a serem enfrentados na APS: condição inadequada da rede física das Unidades Básicas de Saúde (UBS); financiamento ínfimo; dificuldades de integração da APS com a atenção especializada (referência e contra referência); bem como deficiência na incorporação de recursos humanos qualificados (Fausto et al., 2018).

No que se refere às tentativas de mudanças deste cenário, as PIC têm demonstrado que podem promover maior satisfação no trabalho em virtude das mudanças que promovem na organização do trabalho, permitindo maior interação entre práticas e saberes dos profissionais, bem como entre estes e os usuários (Barros, Spadacio, \& Costa, 2018).

A complexidade que se lança ao conceito de saúde atravessa nosso cotidiano enquanto profissionais gestores e usuários de serviços de assistência. Assim, vários sentidos de saúde interessam, já que acreditamos que a partir daí emerge possibilidade para refletir sobre as PIC nos serviços de saúde de Atenção Primária.

O conceito de saúde se destaca por mudanças marcantes na história mundial. De acordo com Scliar (2007), este conceito é reflexo da conjuntura histórica, geográfica, social, econômica, política e cultural da época, uma vez que saúde não simboliza a mesma coisa para todas as pessoas.

Serrão (2010), ao pensar no conceito de saúde proposto pela OMS, "[...] completo bemestar físico, mental e social e não apenas a ausência de doença ou enfermidade" (OMS, 1978), acredita que apesar deste não negar a existência de doença, o mesmo a define como um estado positivo, o qual se descentrou dela, e também dinâmico, que vai se transformando no decorrer da vida dos indivíduos. Não há como estabelecer um estado definido de saúde, dado que este vai se construindo e alcançando individualmente, tratando de uma autopercepção.

Todavia, embora inquestionável o avanço dessa concepção ampliada de saúde, essa conceituação positiva traz um novo problema, conforme aponta Czeresnia e De Freitas (2016), que é a questão da necessidade de promover a vida nas suas múltiplas dimensões. Nestas, envolvem-se, de um lado, mecanismos no campo geral do Estado e, do outro, as 
particularidades e autonomia do indivíduo, a qual não pode ser delegada a responsabilidade de uma área de conhecimento, a priori.

Para Rodrigues e Peixoto Júnior (2014), a saúde tratada por uma abordagem qualitativa, a qual privilegia o sentimento subjetivo que se define no interior de uma relação entre corpo e ambiente, antagoniza-se ao hegemônico modelo biomédico, que inclina na tentativa de definir a saúde quantitativamente. Nesta perspectiva, os autores seguem um pensamento em que os estados de saúde são compatíveis com a situação na qual o vivente surge de forma ativa, relacionando com o ambiente de acordo com o modo de vida que criou, considerando suas experiências anteriores.

É interessante observar que as opiniões dos gestores denotam um certo conhecimento da temática envolvendo a integralidade. Assim, podemos notar que esses gestores a conceituam ao seu modo, de acordo com a sua experiência e vivência profissional. Podemos destacar que eles não se prendem a conceitos definidos e acabados, demonstrando que cada um lê o SUS e o representa à sua maneira por meio da percepção.

Em contrapartida, Silva, Miranda, \& Andrade (2017) alertam para a necessidade de compreender a diversidade do termo, em especial, quando tratamos das práticas das equipes de saúde. As ações precisam estar relacionadas com os princípios da integralidade em uma abordagem multidisciplinar, articulando e dialogando com as diferentes áreas do conhecimento. É evidente a complexidade em torno do termo integralidade, diante de sua pluralidade de interpretações, que vão desde as práticas no cuidado até a articulação do sistema. Desse modo, os autores abordam a necessidade de debates, de modo que os profissionais percebam de forma constante a importância da integralidade na rotina dos serviços, sentindo a diferença quando esta não está presente.

Viegas e Penna (2013) defendem que para a construção da integralidade na prática, faz-se necessário o potencial criativo de cada profissional, de forma integrada com a equipe, objetivando uma ação coordenada para a produção e a promoção do cuidado. Entretanto, o autor afirma que essa estratégia ainda não é uma realidade, posto que a assistência ainda é sustentada no modelo médico-centrado, com foco na doença, gerando uma atenção fragmentada. 
Nessa direção, conforme apontam Da Silva Sanches, Matos, \& Azeredo (2018), podemos destacar a contribuição das PIC, que tem no princípio holístico a fundamentação na busca por compreender o ser humano de maneira integral e vinculada a seu contexto de vida.

O olhar ampliado das PIC acerca da interdisciplinaridade está expresso na concepção original da PNPIC, diante do compromisso com a integralidade da atenção à saúde. No entanto, essa dimensão interdisciplinar parece estar ameaçada, frente ao grande descompasso entre a introdução de novas práticas e ausência de parâmetros técnicos para certificá-las. Aos nossos olhos surge o desafio de consolidar e qualificar as práticas já existentes, só então incidiria novas possibilidades.

Sobre cuidado, integralidade e atenção primária, Carnut (2017) descreve os elementos teórico-conceituais destes, ressaltando a sua articulação e essencialidade na estruturação do sistema de saúde no Brasil. O autor conclui que é possível perceber a importância da sua articulação para um processo de produção da saúde que resgate o ser humano como foco das práticas em saúde.

\section{CONCLUSÕES}

A análise qualitativa nos permitiu apreender os sentidos atribuídos por gestores de unidades de saúde com relação à integralidade, saúde e cuidado. Esses significados não seriam descobertos mecanicamente, como é feito através das frequências e porcentagens da análise quantitativa. Os relatos demonstram ainda que persiste a reprodução de ações e práticas centradas no modelo biomédico, ainda que os gestores reconheçam suas limitações. São fatores como esses que dificultam a implementação e expansão de novos modelos de cuidado, como as PIC. Assim, a priorização da mesma lógica de oferta de serviços, baseadas no persistente modelo tecno-assistencial, enraizados nas concepções da medicina alopática, evidencia uma práxis fragmentada e desarticulada na Atenção Primária à Saúde.

Depreende-se que as PIC seriam melhor compreendidas se os gestores de unidades básicas de saúde tivessem uma formação sanitária, com enfoque na Educação Permanente, na legislação do SUS, contribuindo na efetivação do processo de trabalho da equipe, com prioridade para as necessidades de saúde da população. 
Entretanto, a realidade vivenciada por esses gestores demonstra que a dificuldade de conciliar processo de cuidado e gerenciamento, a falta de capacitação para o gerenciamento, bem como a falta de autonomia e a burocracia, constituem-se em desafios que precisam ser pensados e solucionados, uma vez que esses atores são peças fundamentais para a organização do serviço de saúde. Aponta-se, que a metodologia qualitativa foi importante para alcançar os resultados apresentados, posto que permitiu um debruçar sobre o contexto dos serviços da Atenção Primária à Saúde, de forma dinâmica, interativa e interpretativa. Como limitação deste estudo, aponta-se falhas na fonte de dados CNES, uma vez que não apresenta os mesmos números de oferta de PIC que os registros dos serviços de saúde correspondente. Uma tentativa de reparar tal fato seria a atualização do sistema quanto aos registros da oferta das PIC.

\section{REFERÊNCIAS}

Bardin, L. (2016). Análise de Conteúdo. Tradução: Luís Augusto Pinheiro. São Paulo: Edições 70.

Barros, N. F., Spadacio, C., \& Costa, M. V. (2018). Trabalho interprofissional e as Práticas Integrativas e Complementares no contexto da Atenção Primária à Saúde: potenciais e desafios. Saúde em Debate, 42(spe1), 163-173. https://doi.org/10.1590/0103-11042018s111

Brasil. (1986). Relatório Final da $8^{a}$ Conferência Nacional de Saúde. Recuperado de http://bvsms.saude.gov.br/bvs/publicacoes/8_conferencia_nacional_saude_relatorio_final.pdf. Acesso em: 11 mar. 2020.

Brasil. (2006). Ministério da Saúde. Portaria n. 971, de 3 de maio de 2006. Aprova a Política Nacional de Práticas Integrativas e Complementares no Sistema Único de Saúde. Recuperado de http://bvsms.saude.gov.br/bvs/saudelegis/gm/2006/prt0971_03_05_2006.html. Acesso em: 11 mar. 2020.

Brasil. (2015). Ministério da Saúde. Secretaria de Atenção à Saúde. Departamento de Atenção Básica. Política nacional de práticas integrativas e complementares no SUS: atitude de ampliação de acesso. 2 ed. Brasília.

Brasil. (2017). Ministério da Saúde. Portaria $n^{\circ}$ 2.436, de 21 de setembro de 2017. Recuperado de http://bvsms.saude.gov.br/bvs/saudelegis/gm/2017/prt2436_22_09_2017.html. Acesso em: 11 mar. 2020.

Brasil. (2018). Ministério da Saúde. Portaria n 702, de 21 de março de 2018. Altera a Portaria de Consolidação n²/GM/MS, de 28 de setembro de 2017, para incluir novas práticas na Política Nacional. Diário Oficial da União.

Buss, P. M., \& Carvalho, A. I. (2009). Desenvolvimento da promoção da saúde no Brasil nos últimos vinte anos (1988-2008). Ciência \& Saúde Coletiva, 14(6), 2305-2316. https://doi.org/10.1590/S141381232009000600039

Carnut, Leonardo. (2017). Cuidado, integralidade e atenção primária: articulação essencial para refletir sobre o setor saúde no Brasil. Saúde em Debate, 41(115), 1177-1186. https://doi.org/10.1590/0103-1104201711515.

Czeresnia, D., \& De Freitas, C. M. (2016). Promoção da saúde: conceitos, reflexões, tendências. Rio de Janeiro: Editora Fiocruz. 
Da Silva Sanches, F., De Matos, G. C., \& Azeredo, T. B. (2018). Oferta de práticas integrativas e complementares nas unidades públicas de saúde do município do Rio de Janeiro (2005-2016). VITTALLERevista de Ciências da Saúde, 30(1), 84-97. https://doi.org/10.14295/vittalle.v30i1.7521

Fausto, M. C. R., Rizzoto, M. L. F., Giovanella, L., Seidl, H., Bousquat, A., Almeida, P. F., \& Tomasi, E. (2018). O futuro da Atenção Primária à Saúde no Brasil. Saúde em Debate, 42(spe1), 12-14. https://doi.org/10.1590/0103-11042018s101.

Mozzato, A. R., Grzybovski, D., Teixeira, A. N. (2016). Análise qualitativa nos estudos organizacionais: vantagens no uso do software NVIVO. Revista Alcance, 23(4), 578-587. http://dx.doi.org/10.14210/alcance.v23n4(OutDez).p578-587

Oliveira, M. A. C., Pereira, I. C. (2013). Primary health care essential attributes and the family health strategy. Revista Brasileira de Enfermagem, 66(SPE), 158-164. https://doi.org/10.1590/S0034-71672013000700020.

Organização Mundial de Saúde. (2002). Estrategia de la OMS sobre medicina tradicional 2002-2005. Recuperado de https://apps.who.int/medicinedocs/pdf/s2299s/s2299s.pdf. Acesso em: 11 mar. 2020.

Organização Mundial de Saúde. (1978). Promocíon y desarrollo de la medicina tradicional. Recuperado de https://apps.who.int/medicinedocs/documents/s21404es/s21404es.pdf. Acesso em: 11 mar. 2020.

Pagliosa, F. L., \& Da Ros, M. A. (2008). O relatório Flexner: para o bem e para o mal. Revista Brasileira de Educação Médica, 32(4), 492-499. https://doi.org/10.1590/S0100-55022008000400.

Pinto, H. A. (2014). Múltiplos olhares sobre e a partir do Programa Nacional de Melhoria do Acesso e Qualidade (Dissertação de Mestrado). Universidade Federal do Rio Grande do Sul, Porto Alegre, RS, Brasil.

Rios, E. R. G., Franchi, K. M. B., Silva, R. M., Amorim, R. F., \& Costa, N. C. (2007). Senso comum, ciência e filosofia: elo dos saberes necessários à promoção da saúde. Ciência \& Saúde Coletiva, 12(2), 501509. https://doi.org/10.1590/S1413-81232007000200026

Rodrigues, M., \& Peixoto Júnior, C. A. (2014). Reflexões sobre conceitos afirmativos de saúde e doença nas teorias de Georges Canguilhem e Donald Winnicott. Physis: Revista de Saúde Coletiva, 24, 291-310. https://doi.org/10.1590/S0103-73312014000100016.

Scliar, M. (2007). História do conceito de saúde. Physis: Revista de Saúde Coletiva, 17, $29-41$. https://doi.org/10.1590/S0103-73312007000100003.

Serrão, D. Procurar a sabedoria, partilhar o conhecimento. Porto: Cofanor, 2010.

Silva, M. V. S. D., Miranda, G. B. N., \& Andrade, M. A. D. (2017). Sentidos atribuídos à integralidade: entre o que é preconizado e vivido na equipe multidisciplinar. Interface-Comunicação, Saúde, Educação, 21, 589-599. https://doi.org/10.1590/1807-57622016.0420.

Taquette, S. R., \& Minayo, M. C. (2016). Análise de estudos qualitativos conduzidos por médicos publicados em periódicos científicos brasileiros entre 2004 e 2013. Physis: Revista de Saúde Coletiva, 26, 417-434. https://doi.org/10.1590/S0103-73312016000200005.

Telesi Júnior, E. (2016). Práticas integrativas e complementares em saúde, uma nova eficácia para o SUS. Estudos Avançados, 30(86), 99-112. https://doi.org/10.1590/S0103-40142016.00100007.

Tesser, C. D. (2018). Práticas integrativas e complementares e racionalidades médicas no SUS e na atenção primária à saúde: possibilidades estratégicas de expansão. Journal of Management \& Primary Health Care, 8(2), 216-232. https://doi.org/10.14295/jmphc.v8i2.528.

Viegas, S. M. F., \& Penna, C. M. M. (2013). A construção da integralidade no trabalho cotidiano da equipe saúde da família. Escola Anna Nery, 17(1), 133-141. https://doi.org/10.1590/S1414-81452013000100019. 